\title{
Isothermal nucleic acid amplification in bioanalysis
}

\author{
María Jesús Lobo-Castañón ${ }^{1}$
}

(C) Springer-Verlag Berlin Heidelberg 2016

Chemical sensors and biosensors can provide rapid access to chemical and biological information, and will be a key player for the next technology revolution, where a world of ubiquitous sensors is foreseen. DNA sensors are of particular interest since the detection of specific sequences of nucleic acids is becoming fundamental in a wide range of fields, such as early diagnostics, forensic analysis, or pathogen control. Furthermore, the introduction of synthetic nucleic acids selected by evolutionary molecular biology approaches (aptamers) provides new opportunities for detecting non-nucleic acid targets, including different biomarkers such as small molecules, peptides, proteins, or even whole cells. This fact has dramatically widened the application areas of nucleic acid based sensors.

One major challenge facing DNA-based sensors is the extremely low concentration of targets (nucleic acids or other biomarkers) in real-world problems. In traditional nucleic acid sensors, a 1:1 oligonucleotide receptor/target ratio limits its sensitivity, especially when facing "real samples." In fact, most reported devices are proof-ofprinciple studies that are tested in synthetic samples. Qualification for applications outside an academic laboratory usually requires coupling sensors to the most widely known nucleic acid amplification strategy, polymerase chain reaction (PCR). However, nucleic acid amplification technology has been highly developed since the invention

Published in the topical collection Isothermal Nucleic Acid Amplification in Bioanalysis with guest editor Maria Jesus Lobo Castañón.

María Jesús Lobo-Castañón

mjlc@uniovi.es

1 Departamento de Química-Física y Analítica, Universidad de Oviedo, c/ Julián Clavería, 8, 33006 Oviedo, Spain of PCR. Although PCR has become one of the most valuable tools in virtually all scientific fields, alternative DNA amplification methods have been developed, which eliminate the rapid temperature-cycling demand of PCR; thus simplifying its automation.

Inspired by naturally in vivo DNA replication, researchers have exploited different enzymes such as endonucleases, recombinases, helicases, or strand displacement polymerases to develop various amplification strategies that do not require changing the reaction temperature during the copying process and are extremely fast. It was only recently that many of these strategies were employed to amplify the signal obtained using diverse detection schemes in genosensors and aptasensors, being adapted to different read-out configurations. There are several key challenges to meet before these platforms can find widespread applications. First, copying accuracy is still limited and, in general, these new amplification strategies are prone to false positive results due to nonspecific amplification and primer artefacts. Therefore, improvements in sequencespecific detection strategies or adaptation of new amplification methods are a requisite. Second, the development of simpler solutions, which integrate sample preparation, signal amplification, and detection, must be driven to achieve portable devices useful for point-of-need tests. Increasing amplification efficiency on the surface of different solid supports and reduction of nonspecific adsorption are, in this sense, the main problems to be solved.

A collection of articles in this issue reveals how some of these challenges can be addressed. The combination of advances in isothermal amplification of nucleic acids with new developments in surface chemistry and nanotechnology might turn out to be highly beneficial for further improving the quality of geno- and aptasensors, expanding their range of applications and accelerating their acceptance for solving emerging problems related 
to the detection of DNA and biomarkers. Analytical chemists have the exact knowledge and expertise needed to build on progress in isothermal amplification methods and obtain simple and compact systems for extremely sensitive detection of DNA/RNA, small molecules, proteins, and cells. Hopefully, this topical collection will stimulate interest and new developments in this fascinating field.

I express my sincere appreciation to the authors and reviewers of articles in this Topical Collection. Thanks to the authors for considering this topical collection in Analytical and Bioanalytical Chemistry as the platform for publishing their valuable work and to the reviewers for their contribution in improving the quality of the manuscripts.

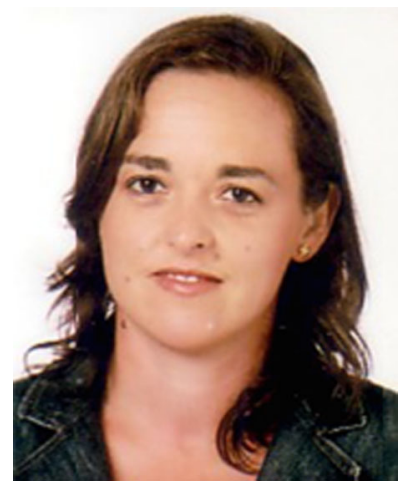

María Jesús Lobo-Castañón holds a $\mathrm{PhD}$ in Chemistry and leads the Electroanalysis Research Group at the Department of Physical and Analytical Chemistry, University of Oviedo, Spain. Since 2001, she has been Professor in Analytical Chemistry at this university. Her research interests focus on the development of electrochemical and SPR sensors for clinical diagnosis and food analysis, using different molecular recognition elements such as enzymes, DNA, aptamers, and molecularly imprinted polymers. She is the author of over 90 articles and book chapters in the field with an $\mathrm{H}$-index of 26. All her written work is available at the group website http://www.unioviedo.es/electroanalisis/. 